\title{
Ichthyosis in Sjögren-Larsson syndrome reflects defective barrier function due to abnormal lamellar body structure and secretion
}

\author{
William B. Rizzo • Dana S'Aulis • M. Anitia Jennings • \\ Debra A. Crumrine $\cdot$ Mary L. Williams • \\ Peter M. Elias
}

Received: 28 September 2009/Revised: 16 December 2009/Accepted: 17 December 2009/Published online: 5 January 2010

(C) The Author(s) 2010. This article is published with open access at Springerlink.com

\begin{abstract}
Sjögren-Larsson syndrome is a genetic disease characterized by ichthyosis, mental retardation, spasticity and mutations in the $A L D H 3 A 2$ gene coding for fatty aldehyde dehydrogenase, an enzyme necessary for oxidation of fatty aldehydes and fatty alcohols. We investigated the cutaneous abnormalities in 9 patients with SjögrenLarsson syndrome to better understand how the enzymatic deficiency results in epidermal dysfunction. Histochemical staining for aldehyde oxidizing activity was profoundly reduced in the epidermis. Colloidal lanthanum perfusion studies showed abnormal movement of tracer into the extracellular spaces of the stratum corneum consistent with a leaky water barrier. The barrier defect could be attributed to the presence of abnormal lamellar bodies, many with disrupted limiting membranes or lacking lamellar contents. Entombed lamellar bodies were present in the cytoplasm of corneocytes suggesting blockade of lamellar body secretion. At the stratum granulosum-stratum corneum interface, non-lamellar material displaced or replaced secreted lamellar membranes, and in the stratum corneum, the number of lamellar bilayers declined and lamellar membrane organization was disrupted by foci of lamellar/nonlamellar phase separation. These studies demonstrate the presence of a permeability barrier abnormality in SjögrenLarsson syndrome, which localizes to the stratum corneum
\end{abstract}

W. B. Rizzo $(\bowtie) \cdot$ D. S'Aulis · M. A. Jennings

Department of Pediatrics, University of Nebraska Medical

Center, 985456 Nebraska Medical Center, Omaha,

NE 68198-5456, USA

e-mail: wrizzo@unmc.edu

D. A. Crumrine · M. L. Williams · P. M. Elias Department of Dermatology, University of California, San Francisco, CA, USA interstices and can be attributed to abnormalities in lamellar body formation and secretion.

Keywords Ichthyosis - Fatty alcohol · Fatty aldehyde . Aldehyde dehydrogenase $\cdot$ Stratum corneum .

Genetic disease

$\begin{array}{ll}\text { Abbreviations } \\ \text { FALDH } & \text { Fatty aldehyde dehydrogenase } \\ \text { LB } & \text { Lamellar body } \\ \text { SC } & \text { Stratum corneum } \\ \text { SG } & \text { Stratum granulosum } \\ \text { SLS } & \text { Sjögren-Larsson syndrome }\end{array}$

\section{Introduction}

Sjögren-Larsson syndrome (SLS) is a rare, autosomal recessive disorder characterized by ichthyosis, mental retardation and spastic diplegia or tetraplegia $[26,35]$. The ichthyosis is usually apparent at birth and often has a mild erythematous appearance that fades over time [21]. A collodion membrane is rarely seen. Pruritus is a common complaint in infants and older patients. Developmental delay and spasticity develop over the first 2 years [12] and white matter changes are typically seen on brain imaging $[36,40]$. Many patients have photophobia and exhibit glistening white dots on the retina [37]. Patients typically survive into adulthood.

Sjögren-Larsson syndrome (SLS) is caused by mutations in the $A L D H 3 A 2$ gene that encodes fatty aldehyde dehydrogenase (FALDH) [2], an enzyme that catalyzes the oxidation of aliphatic aldehydes to fatty acids [17, 29]. 
Despite knowing the enzymatic defect in SLS, the pathogenic mechanisms resulting in ichthyosis have been elusive [27]. FALDH acts on several aliphatic aldehydes, but no single lipid has been identified yet as central to the pathogenesis. Fatty aldehydes and their precursor lipids, such as fatty alcohols [30] and leukotriene B4 [38, 39], accumulate in tissues of SLS patients. In cultured SLS keratinocytes, impaired oxidation of fatty alcohols (C16 and C18) leads to their metabolic diversion into biosynthetic pathways for wax esters and neutral ether glycerolipids, which consequently accumulate [31]. FALDH is also involved in $\omega$ oxidation of very-long-chain fatty acids [32]. These lipid abnormalities, or others yet to be identified in SLS, may contribute to the cutaneous symptoms.

Defects in lipid metabolism underlie many of the ichthyoses, including neutral lipid storage disease with ichthyosis (Chanarin-Dorfman syndrome), X-linked ichthyosis, Refsum disease, Harlequin ichthyosis and several forms of autosomal recessive congenital ichthyosis [7]. Since the lipid composition of the stratum corneum (SC) membranes is critical for maintaining the epidermal water barrier, certain alterations in SC membrane formation or lipid composition appear to provoke a leaky barrier, which in turn 'drives' the ichthyosiform dermatosis [4]. Importantly, with the exception of Harlequin ichthyosis, lamellar body formation and secretion are not impaired. Instead, most of these lipid metabolic disorders have in common lamellar/non-lamellar phase separation as the basis for the permeability barrier abnormality [7]. To date, neither barrier function nor SC membrane formation have been assessed in SLS.

Membranes destined for the SC are synthesized in stratum granulosum (SG) cells and packaged within the transGolgi network into lamellar bodies (LB) [5], which then fuse with the apical plasma membrane of the outermost SG cell, releasing their lamellar contents into the $\mathrm{SC}$ interstices [22]. In normal epidermis, LB secretion results in the transfer of all lamellar body contents to the extracellular spaces, leaving corneocytes devoid of lipids [6]. The deposited LB lipids are subsequently processed into a hydrophobic, equimolar mixture of ceramides, free fatty acids and cholesterol that self-assembles into mature SC membranes. Prior reports of isolated patients with SLS have described membranous lipid inclusions in the cytosol of SG cells and within corneocytes [11,23], as well as structurally abnormal LB [34]. Nevertheless, whether these features are simply disease markers, or are important for the pathogenesis of the epidermal abnormalities in SLS is not known.

Here, we investigated a group of SLS patients with the aim of further defining the link between genetic defect and clinical phenotype by focusing on the functional and structural abnormalities that contribute to ichthyosis. Since the cutaneous phenotype in all other ichthyoses studied to date likely reflects a metabolic response to an underlying permeability barrier abnormality, we began with an assessment of barrier function in SLS, followed by an assessment of the cellular pathogenesis of the barrier abnormality. Our findings indicate that the ichthyosis in SLS is associated with a barrier abnormality that is not only characterized by structurally defective SC lamellar membranes, but also by a global defect in LB formation and secretion.

\section{Materials and methods}

Patients

Sjögren-Larsson syndrome (SLS) patients were diagnosed based on their clinical features, deficient FALDH enzyme activity and the presence of $A L D H 3 A 2$ mutations. Patients $\# 1$ and \#2 are siblings who have been previously reported [20]. Patients \#7 and \#8 are also siblings; other patients are single probands from unrelated families. This human research was approved by the Institutional Review Boards at the University of Nebraska Medical Center, Medical College of Virginia of Virginia Commonwealth University and University of California, San Francisco. Informed consent was obtained from all subjects or, in the case of minors, from their parents.

\section{Mutation analysis}

Genomic DNA was isolated from blood or cultured skin fibroblasts using standard methods. ALDH3A2 exons were amplified by PCR as described [28]. Mutations were detected by directly sequencing the amplified exons using the BigDye Terminator cycle Sequencing Kit (Applied Biosystems, Inc., Foster City, CA) and ABI 377A sequencer. Mutations were identified by comparison to the GenBank sequence of ALDH3A2 (accession NM_000382.1) and designated using the cDNA numbering system.

Enzymatic studies

Cultured skin fibroblasts were established from skin biopsies and grown as described [29]. Cells were harvested and FALDH activity was measured using octadecanal as substrate [29]. Enzyme activity was expressed as pmol octadecanal oxidized/min/mg cell protein.

Light and electron microscopy

Skin punch biopsies were fixed with glutaraldehyde and post-fixed in osmium tetroxide or ruthenium tetroxide, followed by dehydration and embedding in an Epon-epoxy 
mixture or in lipid-retaining resin-white as described [3]. To evaluate permeability barrier dysfunction, the skin biopsies from two SLS patients were immersed in $4 \%$ colloidal lanthanum, a water-soluble electron-dense tracer, for at least $1 \mathrm{~h}$ prior to osmium tetroxide post-fixation and Epon/epoxy embedding as described [5, 33]. Ultrathin sections were examined with a Zeiss $10 \mathrm{~A}$ electron microscope operated at $60 \mathrm{kV}$.

Histochemical staining for aldehyde oxidizing activity

Skin punch biopsies were obtained from the upper arm and immediately frozen in optimal cutting temperature compound using dry ice. The tissues were sectioned at $16 \mu \mathrm{m}$ and kept frozen at $-70^{\circ} \mathrm{C}$ until use. Each slide contained sections of skin from SLS and control subjects. Aldehyde oxidizing activity was detected according to Judge et al. [16] except sections were not acetone-extracted and $8 \mathrm{mM}$ octanal was used as substrate. The slides were incubated at $37^{\circ} \mathrm{C}$ in $50 \mathrm{mM}$ Tris- $\mathrm{HCl}$ buffer, $\mathrm{pH} 8.5$ containing $3.0 \mathrm{mM} \mathrm{NAD}+, 16 \mathrm{mM}$ cobaltous chloride, $0.33 \mathrm{mg} / \mathrm{ml}$ MTT and $8.0 \mathrm{mM}$ octanal. After $1-3 \mathrm{~h}$, the slides were rinsed twice in water for $5 \mathrm{~min}$ each and mounted in glycerin. Slides were examined by light microscopy and photographed.

Detection of apoptotic cells

Cells undergoing apoptosis were detected in skin biopsy sections by TdT-mediated dUTP nick end labeling
(TUNEL) using the In Situ Cell Death Detection Kit (Roche Applied Science, Indianapolis, IN) according to the manufacturer's instructions. TUNEL positive cells in the SG were quantitated by photographing sections at $20 \times$ magnification and counting the stained cells in the field of view.

\section{Results}

We studied 9 SLS patients from 7 unrelated families (Table 1). The patients ranged from 1 year of age to 34 years. All patients had generalized hyperkeratosis and neurologic symptoms that are typical of SLS, along with diagnostic reductions in fibroblast FALDH activity (2-16\% of normal). DNA analysis demonstrated mutations in the $A L D H 3 A 2$ gene in all patients. Eight patients had homozygous mutations, whereas one patient (\#3) was a compound heterozygote who carried one common ALDH3A2 mutation (c.1297_1298delGA) and a presumptive second unidentified mutation (Table 1).

Light microscopy of the skin showed epidermal hyperplasia with prominent hyperkeratosis, papillomatosis and spongiosis (Fig. 1a). The SC was thickened and appeared more dense and compact in regions close to the SG. The granular layer tended to be normal in size (Fig. 1b). Neither the SC nor the SG stained with oil red O, suggesting that large amounts of neutral lipid do not accumulate in SLS (not shown). In one SLS patient examined (patient \#8), the number of SG cells staining positive with TdT-

Table 1 Sjögren-Larsson syndrome patients studied

\begin{tabular}{|c|c|c|c|c|}
\hline Patient & Age (years)/Sex & $\begin{array}{l}\text { Fibroblast FALDH } \\
\text { activity }\left(\% \text { of control }{ }^{\mathrm{c}}\right)\end{array}$ & $A L D H 3 A 2$ genotype & Investigations performed \\
\hline $1^{\mathrm{a}}$ & $32 / \mathrm{M}$ & 5 & $\begin{array}{l}\text { c.798+5_798+6del/ } \\
\text { c.798+5_798+6del }\end{array}$ & EM: $\mathrm{OsO}_{4}, \mathrm{RuO}_{4}^{\mathrm{d}}$ \\
\hline $2^{\mathrm{a}}$ & $34 / \mathrm{F}$ & 4 & $\begin{array}{l}\text { c.798+5_798+6del/ } \\
\text { c. } 798+5 \_798+6 \mathrm{del}\end{array}$ & EM: $\mathrm{OsO}_{4}, \mathrm{RuO}_{4}$ \\
\hline 3 & $5 / \mathrm{M}$ & 9 & c.1297_1298delGA/? & EM: $\mathrm{OsO}_{4}, \mathrm{RuO}_{4}$ \\
\hline 4 & $4 / \mathrm{M}$ & 3 & c. $798+5 \mathrm{G}>\mathrm{A} / \mathrm{c} .798+5 \mathrm{G}>\mathrm{A}$ & EM: $\mathrm{OsO}_{4}, \mathrm{RuO}_{4}$ \\
\hline 5 & $3 / \mathrm{F}$ & 7 & c. $798+1 \mathrm{delG} / \mathrm{c} .798+1 \mathrm{delG}$ & EM: $\mathrm{OsO}_{4}, \mathrm{RuO}_{4}$ \\
\hline 6 & $1 / \mathrm{M}$ & 7 & c. $710 \mathrm{G}>\mathrm{A} / \mathrm{c} .710 \mathrm{G}>\mathrm{A}$ & EM: $\mathrm{OsO}_{4}, \mathrm{RuO}_{4}$ \\
\hline $7^{\mathrm{b}}$ & $21 / \mathrm{M}$ & 13 & $\begin{array}{l}\text { c.1297_1298delGA/ } \\
\text { c.1297_1298delGA }\end{array}$ & Lanthanum perfusion \\
\hline $8^{\mathrm{b}}$ & $19 / \mathrm{F}$ & 16 & $\begin{array}{l}\text { c.1297_1298delGA/ } \\
\text { c.1297_1298delGA }\end{array}$ & Histochemical staining, TUNEL \\
\hline 9 & $15 / \mathrm{F}$ & 2 & c. $710 \mathrm{G}>\mathrm{A} / \mathrm{c} .710 \mathrm{G}>\mathrm{A}$ & Lanthanum perfusion \\
\hline
\end{tabular}

a Siblings in family 1

b Siblings in family 2

${ }^{c}$ Control FALDH activity was $8,860 \pm 1,624 \mathrm{pmol} / \mathrm{min} / \mathrm{mg}$ protein $(n=22)$

${ }^{\mathrm{d}}$ Electron microscopy with osmium tetroxide and ruthenium tetroxide post-fixation 
mediated dUTP nick end labeling (TUNEL), a marker for apoptosis, were increased by $80 \%$ compared to a normal control (SLS: $18 \pm 3$ cells/field, $n=7$; control: $10 \pm 3$ cells/field, $n=7$ ).

Aldehyde oxidizing activity in skin was visualized using a histochemical staining method with octanal as substrate (Fig. 2). Normal skin showed abundant staining of the epidermis and there was scattered staining of dermal fibroblasts (Fig. 2a). The SC displayed no enzyme activity. Staining was dependent on the presence of octanal (Fig. 2c) and was completely inhibited by the addition of $10 \mathrm{mM}$ disulfiram, which is an aldehyde dehydrogenase inhibitor (data not shown). In contrast to normal skin, SLS skin showed a profound lack of enzyme activity throughout the epidermis and dermis (Fig. 2b).

To evaluate the integrity of the epidermal water barrier in SLS, we perfused freshly obtained skin biopsies from 2 SLS patients (patients \#7 and \#9) with colloidal lanthanum nitrate prior to $\mathrm{OsO}_{4}$ post-fixation. Lanthanum is a lowmolecular weight, water-soluble, electron-dense tracer that is normally excluded from entry into the SC. In the SLS skin samples, however, the tracer moved outward beyond the SG, and entered the SC interstices (Fig. 3). Since tracer did not enter corneocytes, a defect in corneocyte integrity can be excluded as the cause of the permeability barrier abnormality in SLS. These findings indicate accelerated, paracellular traverse of water, with further localization of the permeability barrier abnormality to the SC extracellular domains.

Next we assessed the cellular basis for the permeability barrier abnormality in SLS. Skin biopsies from 6 SLS patients (patients \#1-\#6) were examined by electron microscopy using standard methods with post-fixation in osmium tetroxide and also ruthenium tetraoxide, which allows better visualization of SC lipid membranes. The SLS skin revealed normal numbers (i.e., density) of LB in the SG, but individual organelles exhibited several structural abnormalities. Some LB appeared empty or contained non-lamellar contents, whereas many others displayed disrupted or absent limiting membranes (Fig. 4). The latter feature has not yet been described in other lipid metabolic disorders associated with ichthyosis [7]. At the SG-SC interface, non-lamellar material displaced or replaced the normal lamellar contents (Fig. 5). Unsecreted LB accumulated at the outer periphery of SG cells and became entombed in the corneocyte cytosol (Figs. 6, 7). This finding suggests that secretion of LB at the SG-SC border is impaired, due to defective LB fusion with the outer SG
Fig. 1 Histopathology of SLS. a At low magnification, epidermal hyperplasia, spongiosis, and prominent hyperkeratosis are evident in patient \#4. Note compactness of lower SC (solid arrow) and loosely organized mid- to upperSC (open arrows). b At higher magnification, the granular layer (arrows) is normal in size. Epon embedded, $1 \mu \mathrm{m}$ section, toluidine blue staining

Fig. 2 Histochemical staining for aldehyde (octanal) oxidizing activity in normal and SLS skin. a Normal skin shows abundant staining of the epidermis, but not of the SC. b SLS skin (patient \#8) has a profound reduction in enzyme activity in the epidermis. c Control showing normal skin incubated without octanal
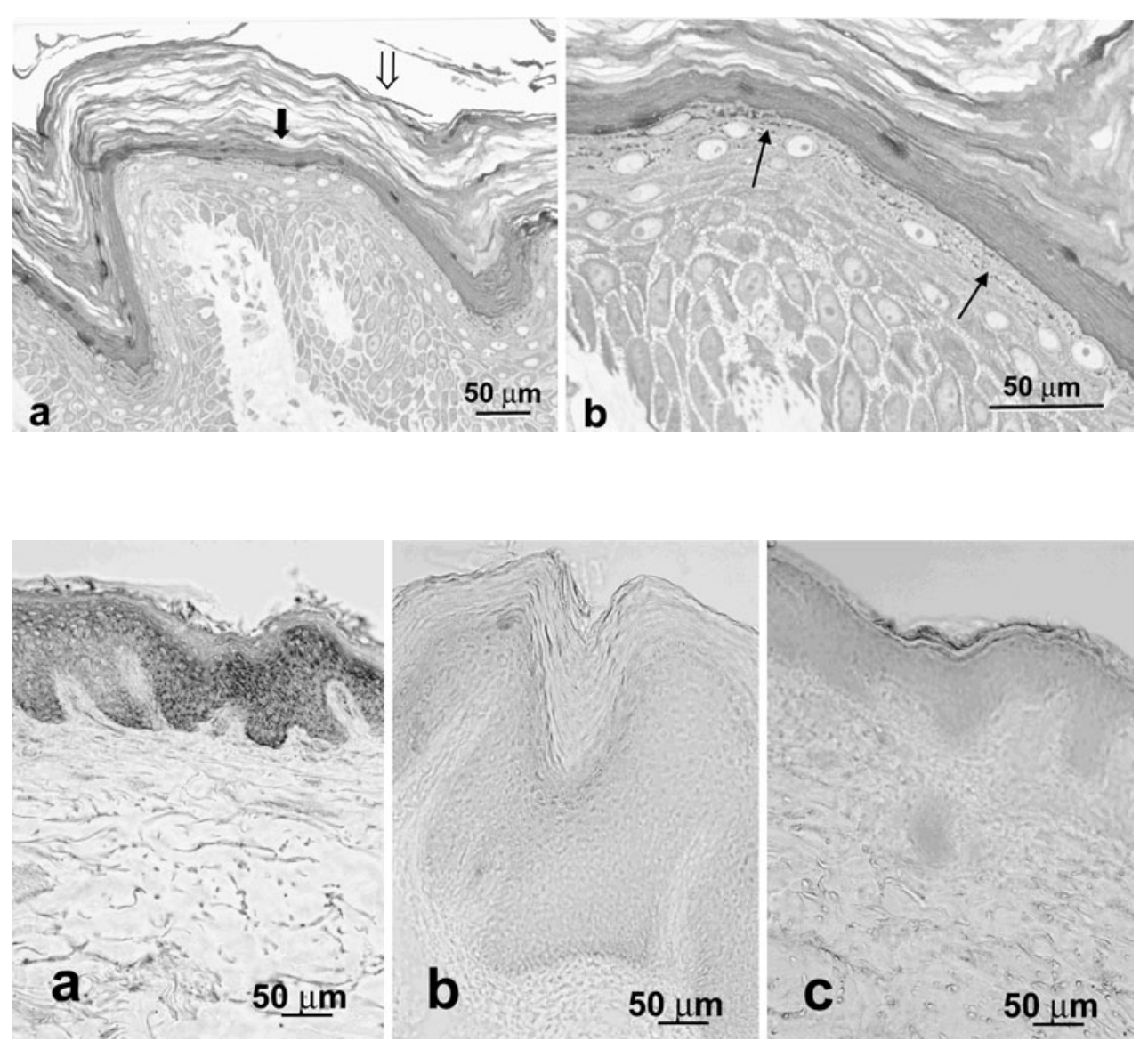


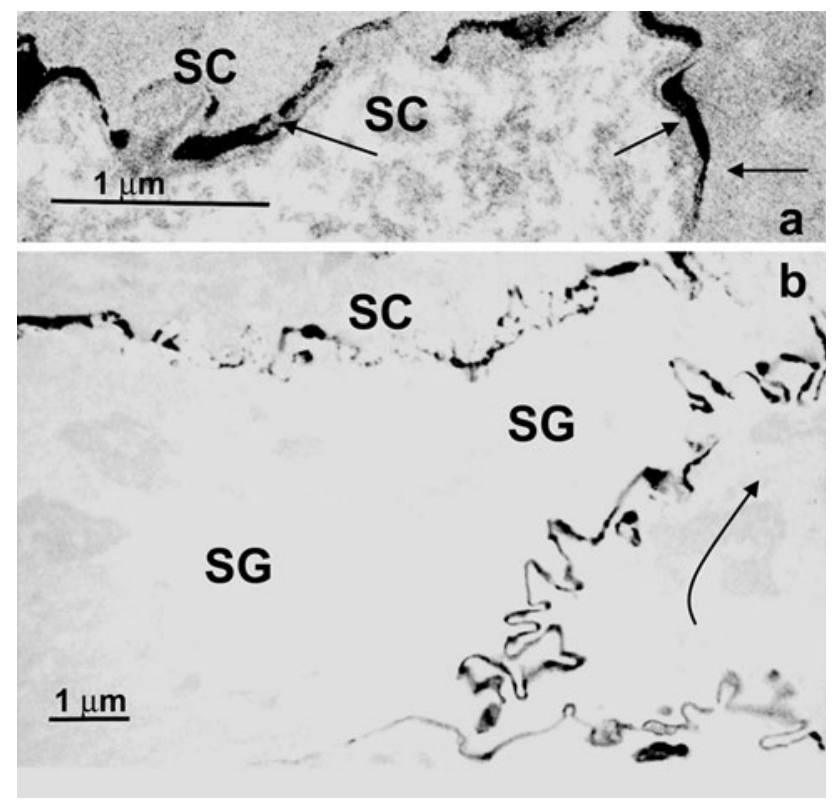

Fig. 3 Lanthanum perfusion demonstrates a leaky epidermal water barrier in SLS (patient \#7). a Lanthanum tracer, which reflects the pathway of water movement and is completely excluded from the SC in normal skin, but breaches the SC via the extracellular spaces in SLS (arrows). b Tracer moves outward through SG (curved arrow) and remains restricted to SC interstices (a, arrows). a, b, osmium tetroxide and ruthenium tetroxide post-fixation

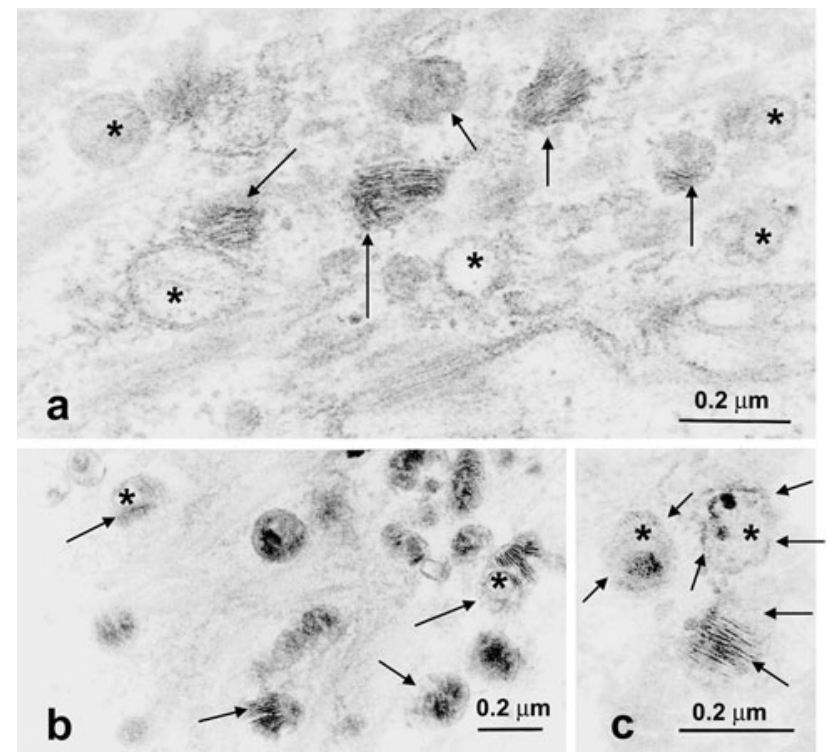

Fig. 4 Abnormal lamellar bodies in SLS. a-c Although the number (density) of lamellar bodies appears normal in SLS, many organelles appear empty (asterisks) or display non-lamellar contents. Moreover, the limiting membrane of many individual organelles appears disrupted or absent (arrows). Osmium tetroxide post-fixation. a, c Patient \#4. b Patient \#3

plasma membrane. In the SC, lamellar domains were interspersed with lacunae filled with non-lamellar material, indicating lamellar/non-lamellar phase separation

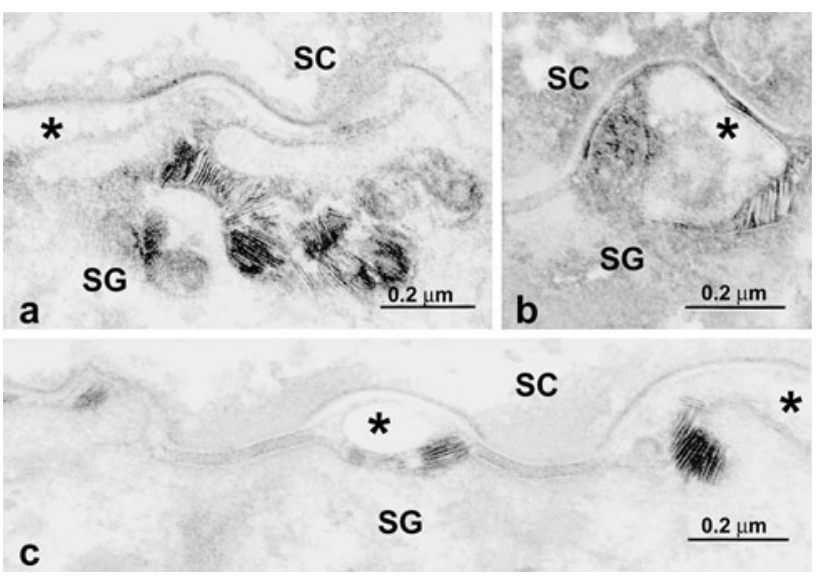

Fig. 5 Secretion of non-lamellar contents at stratum corneum (SC) stratum granulosum (SG) interface in SLS (patient \#4). a-c Much of the SG-SC interface is occupied by non-lamellar contents (asterisks) that displace or replace secreted lamellar material. However, the cornified lipid envelope appears normal. Osmium tetroxide postfixation

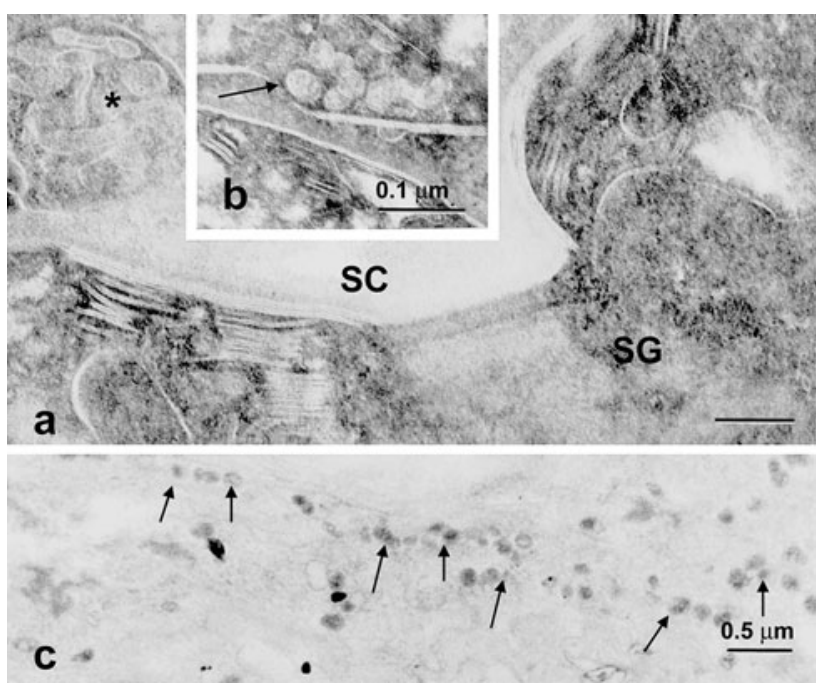

Fig. 6 Abnormal lamellar body secretion in SLS results in entombment of organelle contents in corneocytes. a, b Unsecreted organelles become entombed in the corneocyte cytosol (a asterisks; $\mathbf{b}$ arrow) of patient \#4. c Note the increased concentration of unsecreted lamellar bodies (arrows) at the periphery of outer SG cells in patient \#3. a, b, ruthenium tetroxide post-fixation; $\mathbf{c}$ osmium tetroxide post-fixation

(Fig. 7a), a structural feature of other lipidoses associated with ichthyosis [7]. The structurally abnormal LB and failure of LB secretion were further associated with a paucity of lamellar bilayers in the SC (Fig. 7b). In contrast, the cornified lipid envelope appeared normal (Fig. 5a, c).

The structural abnormalities in SG and SC were observed in all SLS patients studied. There was no apparent relationship between the severity of the structural abnormalities and age of the patient, $A L D H 3 A 2$ genotype or residual enzyme activity measured in fibroblasts. 

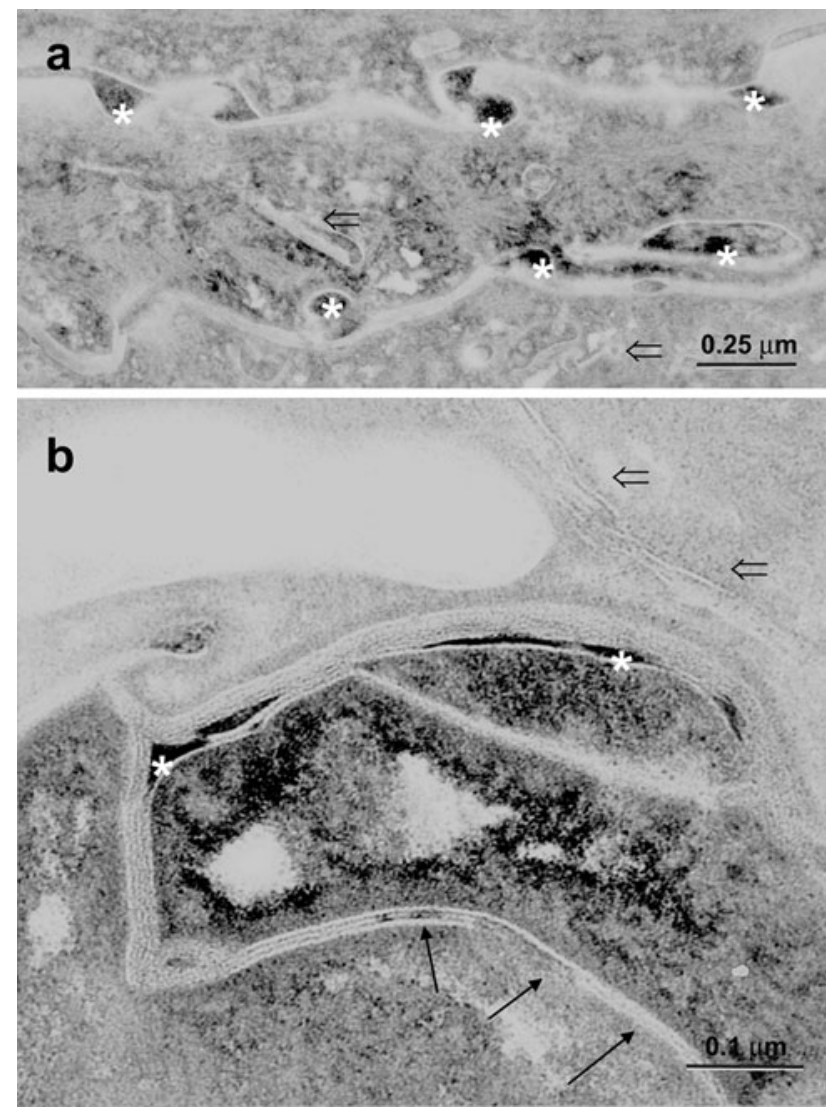

Fig. 7 Decreased lamellar bilayers and lamellar/non-lamellar phase separation in SC interstices of patient \#4. a, b Entombed lamellar contents in corneocyte cytosol is evident (open arrows). Lamellar domains are interspersed with lacunae filled with non-lamellar material (asterisks). b Blockade of secretion (c.f., Fig. 6) also results in paucity of lamellar bilayers (arrows). a, b ruthenium tetroxide post-fixation

\section{Discussion}

Although a defective epidermal water barrier has been presumed to exist in SLS, we provide the first experimental evidence for a barrier abnormality; i.e., the demonstration of unimpeded paracellular traverse of colloidal lanthanum between SC corneocytes. The basis for the barrier abnormality is likely twofold. First, our studies demonstrate a widespread disruption in the formation and secretion of LB in the epidermis of SLS patients. The striking abnormalities in LB structure included empty vesicles, inclusion of amorphous material instead of the usual stacked membranes and the presence of LB that have disrupted, or even absent, vesicle membranes. The latter finding suggests that the organelle limiting membrane is damaged after LB has packaged their lipid and non-lipid contents. We interpret our finding of unsecreted LB at the outer periphery of SG cells and the entombment of LB in the cytoplasm of corneocytes to arise from defective vesicle-plasma membrane fusion, perhaps reflecting defects in the organelle limiting membrane. The second reason for the putative barrier abnormality is the paucity and disorganization of SC lamellar membranes, a previously unrecognized structural feature of SLS that was revealed with ruthenium tetroxide post-fixation. Decreased lamellar arrays correlate with the impairment in LB secretion, noted above. But the lamellar domains in the SC are interspersed with lacunae filled with non-lamellar material, which reflects a process of lamellar/ non-lamellar phase separation in the SC similar to several other forms of ichthyosis associated with defective lipid metabolism [7]. Lamellar/non-lamellar phase separation likely also reflects the lipid metabolic abnormality; i.e., the critical ratio of cholesterol, free fatty acids and ceramides is altered sufficiently to prevent incorporation of all secreted lipids within lamellar arrays.

The ichthyosis in SLS is associated with a hyperproliferative epidermal state as demonstrated by radioactive thymidine incorporation [13] and our finding of an increased number of apoptotic cells in the SG.

The ultrastructural abnormalities that we observed were present in all 6 SLS patients examined. We found no relationship between the severity of structural abnormalities in the skin and the patients' ages. Indeed, the clinical appearance of the ichthyosis in SLS does not typically change after infancy. Nor did the structural abnormalities correlate with $A L D H 3 A 2$ genotype or residual enzyme activity in fibroblasts. Most mutations are completely destructive to FALDH catalytic activity [28], and the variation in residual enzyme activity measured in cultured fibroblasts largely reflects the presence of other aldehyde dehydrogenase enzymes that have some ability to oxidize long-chain fatty aldehydes [26].

Defective LB vesicle membranes and impaired LB secretion have not been previously recognized in SLS, but ultrastructural abnormalities have been reported. Similar to our patients here, Shibaki et al. [34] found empty LB, and the presence of irregular granular material and lipid vacuoles in the intercellular domain between corneocytes in 2 Japanese SLS siblings with $A L D H 3 A 2$ mutations. In an atypical 5-year-old Japanese patient with low-normal intelligence, mild neurologic symptoms and unusually late onset of ichthyosis at 1 year of age, Ito et al. [11] found membranous lipid inclusions in the cytoplasm of spinous and granular cells and in corneocytes. Some LB appeared misshapen and contained fine granular or globular contents. This patient was subsequently confirmed to have FALDH deficiency (Rizzo, unpublished). Membranous cytoplasmic inclusions in corneocytes were observed in 2 additional patients who were clinically diagnosed with SLS, but did not have enzymatic or genetic confirmation of the diagnosis [23].

The LB abnormalities seen in the SG, together with the paucity of SC membranes and presence of lacunae 
containing non-membrane material are consistent with a defect in lipid metabolism, but the precise lipids responsible for the cutaneous pathology remain to be identified [27]. Several candidate lipids may have specific deleterious effects at different points in SC membrane formation, i.e., early in LB vesicle membrane formation, packaging of LB membranes destined for the $\mathrm{SC}$, or at the point of $\mathrm{LB}$ exocytosis. Straight-chain fatty alcohols (C16:0 and C18:0), wax esters and neutral ether glycerolipids (alkyldiacylglycerol) accumulate in cultured SLS keratinocytes [31]. These lipids are non-polar and their accumulation in granulosum cells could interfere at any point in LB formation and secretion, or contribute to the lamellar/nonlamellar phase separation seen in the SC. Failure to detect histological evidence of neutral lipid accumulation by oil red $\mathrm{O}$ staining of the SLS skin may reflect dispersion of these neutral lipids in SG and SC membranes or insufficient sensitivity of the stain. Oil red $\mathrm{O}$ staining is usually reserved for ichthyoses associated with accumulation of cytoplasmic lipid droplets, i.e., neutral lipid storage disease and Refsum disease. Alternately, other more polar lipids may be responsible for the epidermal dysfunction. Oxidation of isoprenoid alcohols, such as farnesol and geranylgeraniol, has recently been found to be defective in cultured skin fibroblasts and in the epidermis of SLS patients (Roullet J-B, Steiner R, Rizzo WB, unpublished data). Farnesol induces keratinocyte differentiation in vitro via a PPAR- $\alpha$ dependent mechanism [9]. It also impacts cholesterol biosynthesis by hastening degradation of HMGCoA reductase [24] and inhibiting mevalonate kinase [10], which raises the possibility that biosynthesis of epidermal cholesterol, a major lipid of SC membranes, may be reduced in SLS. Rodents treated with topical HMG-CoA reductase inhibitors, such as lovastatin, exhibit abnormal LB structures, including empty LB, and a defective epidermal water barrier [8, 25]. FALDH is also involved in the $\omega$-oxidation of at least 2 additional lipids: very-long-chain fatty acids [32] and the inflammatory mediator leukotriene B4 [38, 39]. Accumulation of leukotriene B4 seems to contribute to the pruritus seen in SLS [38], but is unlikely to affect the epidermal water barrier.

Recently, it has been speculated that FALDH might be involved in metabolism of $12 R$-eicosanoids derived from arachidonic acid, a lipid pathway that has emerged as critical for normal epidermal function. Two genes in this pathway, $A L O X 12$ and $e L O X 3$, encode enzymes that sequentially metabolize arachidonic acid to the epoxyalcohol 12R-hepoxilin A3 [1]. Mutations in ALOX12 and eLOX3 are responsible for two forms of autosomal recessive congenital ichthyosis, which strongly implies that $12 R$ hepoxilin A3 or its downstream metabolite(s) are necessary for normal barrier function [15]. Furthermore, mutations in two additional genes (CYP4F22 and ichthyin) that encode a putative $\omega$-hydroxylase enzyme and a putative membrane receptor for $12 R$-epoxyalcohols, respectively, also cause ichthyosis [18, 19]. Since the epoxyalcohols are metabolized via $\omega$-hydroxylation to $\omega$-carboxy-acids, it is possible that FALDH is necessary for their oxidation to biologically active products and the ichthyosis in SLS may originate from a block in this step [18].

Other pathogenic mechanisms associated with FALDH deficiency may co-exist. Aliphatic aldehydes have the propensity to react with molecules containing primary amines, such as amino-containing lipids and lysine residues in proteins. In SLS fibroblasts, long-chain aldehydes form covalent Schiff base adducts with phosphatidylethanolamine (PE) generating $N$-alkyl-phosphatidylethanolamine [14]. The addition of the hydrophobic alkyl group to the more polar ethanolamine moiety of PE is likely to perturb its metabolism or proper incorporation into membrane bilayers.

In summary, our findings prompt us to hypothesize that the cutaneous pathogenesis of SLS originates from lipid abnormalities in the SG that disrupt the normal formation and secretion of LB, which consequently leads to intercellular non-membranous lipid deposits in the SC, diminished SC membrane arrays and a defective water barrier. Since the severity of cutaneous disease does not correlate with neurologic symptoms in SLS [12], the pathogenic mechanisms operating in the skin are almost certainly distinct from those in the nervous system. Effective therapeutic approaches in SLS will therefore likely depend on impacting those pathogenic mechanisms that are uniquely critical for each organ.

Acknowledgments This research was supported by NIH grants AR044552, AR019098 and AR39448(PP) from the National Institute of Arthritis and Musculoskeletal and Skin Diseases of the NIH; the Sjögren-Larsson Syndrome Research Fund at the University of Nebraska Foundation; and The Foundation for Ichthyosis and Related Skin Types.

Conflict of interest statement The authors have no conflicts of interest.

Open Access This article is distributed under the terms of the Creative Commons Attribution Noncommercial License which permits any noncommercial use, distribution, and reproduction in any medium, provided the original author(s) and source are credited.

\section{References}

1. Brash AR, Yu Z, Boeglin WE, Schneider C (2007) The hepoxilin connection in the epidermis. FEBS J 274:3494-3502

2. De Laurenzi V, Rogers GR, Hamrock DJ, Marekov LN, Steinert PM, Compton JG, Markova N, Rizzo WB (1996) Sjögren-Larsson syndrome is caused by mutations in the fatty aldehyde dehydrogenase gene. Nat Genet 12:52-57 
3. Demerjian M, Crumrine DA, Milstone LM, Williams ML, Elias PM (2006) Barrier dysfunction and pathogenesis of neutral lipid storage disease with ichthyosis (Chanarin-Dorfman syndrome). J Invest Dermatol 126:2032-2038

4. Elias PM (1983) Epidermal lipids, barrier function, and desquamation. J Invest Dermatol 80(Suppl):44s-49s

5. Elias PM, Cullander C, Maura T, Rassner U, Kömüves L, Brown BE, Menon GK (1998) The secretory granular cell: the outermost granular cell as a specialized secretory cell. J Invest Dermatol Symp Proc 3:87-100

6. Elias PM, Menon GK (1991) Structural and lipid biochemical correlates of the epidermal permeability barrier. Adv Lipid Res 24:1-26

7. Elias PM, Williams ML, Holleran WM, Jiang YJ, Schmuth M (2008) Pathogenesis of permeability barrier abnormalities in the ichthyoses: inherited disorders of lipid metabolism. J Lipid Res 49:697-714

8. Feingold KR, Man MQ, Proksch E, Menon GK, Brown BE, Elias PM (1991) The lovastatin-treated rodent: a new model of barrier disruption and epidermal hyperplasia. J Invest Dermatol 96:201209

9. Hanley K, Kömüves LG, Ng DC, Schoonjans K, He SS, Lau P, Bikle DD, Williams ML, Elias PM, Auwerx J, Feingold KR (2000) Farnesol stimulates differentiation in epidermal keratinocytes via PPARalpha. J Biol Chem 275:11484-11491

10. Hinson DD, Chambliss KL, Toth MJ, Tanaka RD, Gibson KM (1997) Post-translational regulation of mevalonate kinase by intermediates of the cholesterol and nonsterol isoprene biosynthetic pathways. J Lipid Res 38:2216-2223

11. Ito M, Oguro K, Sato Y (1991) Ultrastructural study of the skin in Sjögren-Larsson syndrome. Arch Dermatol Res 283:141-148

12. Jagell S, Heijbel J (1982) Sjögren-Larsson syndrome: physical and neurological features. A survey of 35 patients. Helv Paediatr Acta 37:519-530

13. Jagell S, Lidén S (1982) Ichthyosis in the Sjögren-Larsson syndrome. Clin Genet 21:243-252

14. James PF, Zoeller RA (1997) Isolation of animal cell mutants defective in long-chain fatty aldehyde dehydrogenase. Sensitivity to fatty aldehydes and Schiff's base modification of phospholipids: implications for Sjögren-Larsson syndrome. J Biol Chem 272:23532-23539

15. Jobard F, Lefevre C, Karaduman A, Blanchet-Bardon C, Emre S, Weissenbach J, Ozgüc M, Lathrop M, Prud'homme JF, Fischer J (2002) Lipoxygenase-3 (ALOXE3) and 12(R)-lipoxygenase (ALOX12B) are mutated in non-bullous congenital ichthyosiform erythroderma (NCIE) linked to chromosome 17p13.1. Hum Mol Genet 11:107-113

16. Judge MR, Lake BD, Smith VV, Besley GT, Harper JI (1990) Depletion of alcohol (hexanol) dehydrogenase activity in the epidermis and jejunal mucosa in Sjögren-Larsson syndrome. J Invest Dermatol 95:632-634

17. Kelson TL, Secor M Jr, Rizzo WB (1997) Human liver fatty aldehyde dehydrogenase: microsomal localization, purification, and biochemical characterization. Biochim Biophys Acta 1335:99-110

18. Lefevre C, Bouadjar B, Ferrand V, Tadini G, Megarbane A, Lathrop M, Prud'homme JF, Fischer J et al (2006) Mutations in a new cytochrome $\mathrm{P} 450$ gene in lamellar ichthyosis type 3 . Hum Mol Genet 15:767-776

19. Lefevre C, Bouadjar B, Karaduman A, Jobard F, Saker S, Ozguc M, Lathrop M, Prud'homme JF, Fischer J (2004) Mutations in ichthyin a new gene on chromosome $5 \mathrm{q} 33$ in a new form of autosomal recessive congenital ichthyosis. Hum Mol Genet 13:2473-2482

20. Levisohn D, Dintiman B, Rizzo WB (1991) Sjögren-Larsson syndrome. Case reports. Pediatr Dermatol 8:217-220
21. Liden S, Jagell S (1984) The Sjögren-Larsson syndrome. Int J Dermatol 23:247-253

22. Madison KC (2003) Barrier function of the skin: "la raison d'etre" of the epidermis. J Invest Dermatol 121:231-241

23. Matsuoka LY, Kousseff BG, Hashimoto K (1982) Studies of the skin in Sjögren-Larsson syndrome by electron microscopy. Am J Dermatopathol 4:295-301

24. Meigs TE, Roseman DS, Simoni RD (1996) Regulation of 3hydroxy-3-methylglutaryl-coenzyme A reductase degradation by the nonsterol mevalonate metabolite farnesol in vivo. J Biol Chem 271:7916-7922

25. Menon GK, Feingold KR, Mao-Qiang M, Schaude M, Elias PM (1992) Structural basis for the barrier abnormality following inhibition of HMG CoA reductase in murine epidermis. J Invest Dermatol 98:209-219

26. Rizzo WB (2001) Sjögren-Larsson syndrome: fatty aldehyde dehydrogenase deficiency. In: Scriver CR, Beckman K, Small GM, Valle D (eds) The metabolic \& molecular bases of inherited disease, vol 2. McGraw-Hill, New York, pp 2239-2258

27. Rizzo WB (2007) Sjögren-Larsson syndrome: molecular genetics and biochemical pathogenesis of fatty aldehyde dehydrogenase deficiency. Mol Genet Metab 90:1-9

28. Rizzo WB, Carney G, Lin Z (1999) The molecular basis of Sjögren-Larsson syndrome: mutation analysis of the fatty aldehyde dehydrogenase gene. Am J Hum Genet 65:1547-1560

29. Rizzo WB, Craft DA (1991) Sjögren-Larsson syndrome. Deficient activity of the fatty aldehyde dehydrogenase component of fatty alcohol: NAD+ oxidoreductase in cultured fibroblasts. J Clin Invest 88:1643-1648

30. Rizzo WB, Craft DA (2000) Sjögren-Larsson syndrome: accumulation of free fatty alcohols in cultured fibroblasts and plasma. J Lipid Res 41:1077-1081

31. Rizzo WB, Craft DA, Somer T, Carney G, Trafrova J, Simon M (2008) Abnormal fatty alcohol metabolism in cultured keratinocytes from patients with Sjögren-Larsson syndrome. J Lipid Res 49:410-419

32. Sanders RJ, Ofman R, Dacremont G, Wanders RJ, Kemp S (2008) Characterization of the human omega-oxidation pathway for omega-hydroxy-very-long-chain fatty acids. FASEB J 22:2064-2071

33. Schmuth M, Yosipovitch G, Williams ML, Weber F, Hintner H, Ortiz-Urda S, Rappersberger K, Crumrine D, Feingold KR, Elias PM (2001) Pathogenesis of the permeability barrier abnormality in epidermolytic hyperkeratosis. J Invest Dermatol 117:837-847

34. Shibaki A, Akiyama M, Shimizu H (2004) Novel ALDH3A2 heterozygous mutations are associated with defective lamellar granule formation in a Japanese family of Sjögren-Larsson syndrome. J Invest Dermatol 123:1197-1199

35. Sjögren T, Larsson T (1957) Oligophrenia in combination with congenital ichthyosis and spastic disorders. Acta Psychiatr Neurol Scand 32(Suppl 113):1-113

36. van Domburg PHMF, Willemsen MAAP, Rotteveel JJ, de Jong JG, Thijssen HOM, Heerschap A, Cruysberg JRM, Wanders RJA, Gabreëls FJM, Steijlen PM (1999) Sjögren-Larsson syndrome: clinical and MRI/MRS findings in FALDH-deficient patients. Neurology 52:1345-1352

37. Willemsen MAAP, Cruysberg JRM, Rotteveel JJ, Aandekerk AL, van Domburg PHMF, Deutman AF (2000) Juvenile macular dystrophy associated with deficient activity of fatty aldehyde dehydrogenase in Sjögren-Larsson syndrome. Am J Ophthalmol 130:782-789

38. Willemsen MAAP, Lutt MA, Steijlen PM, Cruysberg JR, van der Graaf M, Nijhuis-van der Sanden MW, Pasman JW, Mayatepek E, Rotteveel JJ (2001) Clinical and biochemical effects of 
zileuton in patients with the Sjögren-Larsson syndrome. Eur $\mathrm{J}$ Pediatr 160:711-717

39. Willemsen MAAP, Rotteveel JJ, de Jong JG, Wanders RJA, Ijlst L, Hoffmann GFM, Mayatepek E (2001) Defective metabolism of leukotriene B4 in the Sjögren-Larsson syndrome. J Neurol Sci 183:61-67
40. Willemsen MAAP, van der Graaf M, van der Knaap MS, Heerschap A, van Domburg PHMF, Gabreels FJ, Rotteveel JJ (2004) MR imaging and proton MR spectroscopic studies in Sjögren-Larsson syndrome: characterization of the leukoencephalopathy. AJNR Am J Neuroradiol 25:649-657 